\title{
Naphthoquinone enhances antigen-related airway inflammation in mice
}

\author{
K. Inoue*, H. Takano*, K. Hiyoshi" ${ }^{\#}$ T. Ichinose ", K. Sadakane ", R. Yanagisawa*, \\ S. Tomura\# ${ }^{\#}$ and Y. Kumagai ${ }^{+}$
}

ABSTRACT: The current authors have previously demonstrated that diesel exhaust particles (DEP) enhance antigen-related airway inflammation in mice. Furthermore, a recent study has shown that organic chemicals in DEP, rather than their carbonaceous nuclei, are important contributors to the aggravating effects of airway inflammation. However, the components in DEP responsible for the enhancing effects on the model remain to be identified.

The current authors investigated the effects of naphthoquinone (NQ), one of the extractable chemical compounds of DEP, on antigen-related airway inflammation, local expression of cytokine proteins, and antigen-specific immunoglobulin (Ig) production in mice. Pulmonary exposure to NQ dose-dependently aggravated antigen-related airway inflammation, as characterised by infiltration of eosinophils and lymphocytes around the airways and an increase in goblet cells in the bronchial epithelium. Combined exposure to NQ and antigen enhanced the local expression of interleukin (IL)-4, IL-5, eotaxin, macrophage chemoattractant protein-1 and keratinocyte chemoattractant, compared with exposure to antigen or NQ alone. Also, NQ exhibited adjuvant activity for the antigen-specific production of $\operatorname{lgG}_{1}$ and $\operatorname{lgG}_{2 a}$.

These results provide the first experimental evidence that naphthoquinone can enhance antigen-related airway inflammation in vivo, and that naphthoquinone can, to some extent, partly play a role in the pathogenesis of diesel exhaust particle toxicity on the condition.

KEYWORDS: Airway inflammation, antigen, immunoglobulin, naphthoquinone

D iesel exhaust particles (DEP) are major contributors to atmospheric particulate air pollution in metropolitan areas. DEP have been correlated to lung cancer, pulmonary fibrosis, chronic alveolitis [1] and oedematous changes [2]. Also, DEP have been implicated to modulate allergic reactions [3, 4]. DEP enhance the antigen-specific immunoglobulin $(\mathrm{Ig}) \mathrm{E}$ response [5, 6] and aggravate airway inflammation induced by repetitive intratracheal instillation of antigen in vivo [7-10].

DEP are complicated particles consisting of carbonaceous nuclei and a vast number of organic chemical compounds such as polyaromatic hydrocarbons, aliphatic hydrocarbons, heterocycles, and quinones. Previous studies have indicated that organic chemicals extracted from DEP result in induction of apoptosis [11], increase oxidative stress [12], and induce the production of inflammatory cells [13-15] through the release of pro-inflammatory molecules in vitro $[15,16]$. The current authors have recently demonstrated that extracted organic chemicals from DEP, rather than residual carbonaceous nuclei of DEP after extraction, predominantly enhance antigen-related airway inflammation in mice [17]. However, detailed studies concerning the component(s) of DEP responsible for their effects on the respiratory system and/or pulmonary diseases remain unsatisfied, which is particularly the case for in vivo studies.

A variety of quinones have been identified as DEP components [18, 19]. Quinones themselves have toxicological properties, serving as alkylating agents and interacting with, for example, flavoproteins to generate reactive oxygen species (ROS) which can induce biological injury [20-23]. Phenanthraquinone (PQ) is one of the quinones contained in DEP $[19,23]$. The current authors have recently shown that PQ induces recruitment of inflammatory cells, such as eosinophils and neutrophils, into the lung with the lung expression of pro-inflammatory molecules such as interleukin (IL)-5 and eotaxin in vivo [24]. More recently, it has also been demonstrated that PQ aggravates antigen-related airway inflammation in mice, and that PQ also has adjuvant activity for antigenspecific Igs [25]. These studies suggest that quinones may be key compounds in the enhancing effects of DEP on allergic airway diseases.
AFFILIATIONS

*Environmental Health Sciences

Division, National Institute for

Environmental Studies,

\#Graduate School of Comprehensive Human Sciences,

+Dept of Environmental Medicine, Institute of Community Medicine, University of Tsukuba

Ibaraki, and

"Dept of Health Science, Oita University of Nursing and Health Science

Oita, Japan.

CORRESPONDENCE

H. Takano

Environmental Health Sciences Division

National Institute for Environmental

Studies

16-2 Onogawa

Tsukuba

305-8506

Japan

Fax: 81298502334

E-mail: htakano@nies.go.jp

Received:

March 082006

Accepted after revision:

October 172006

STATEMENT OF INTEREST

None declared.

European Respiratory Journal Print ISSN 0903-1936 Online ISSN 1399-3003 
Naphthoquinone (NQ) is another extractable compound from DEP [25]. NQ contributes to DEP toxicity through the reduction of superoxide dismutase activity in vitro [26], which raises the possibility that NQ may facilitate airway inflammatory conditions in vivo. The aim of the present study was to elucidate the effects of NQ on airway inflammation, the local expression of cytokines such as IL-4 and IL-5 and chemokines such as eotaxin, macrophage chemoattractant protein (MCP)-1, and keratinocyte chemoattractant $(\mathrm{KC})$ and $\mathrm{Ig}$ production related to antigen exposure.

\section{METHODS}

\section{Animals}

Male ICR mice aged 6-7 weeks and weighing 29-33 g (Japan Clea Co., Tokyo, Japan) were used in all experiments. They were fed a commercial diet (Japan Clea Co.) and given water ad libitum. Mice were housed in an animal facility that was maintained at $24-26^{\circ} \mathrm{C}$ with $55-75 \%$ humidity and a 12 -h light/ dark cycle. The studies adhered to the National Institutes of Health guidelines for the experimental use of animals. All animal studies were approved by the institutional review board of the National Institute for Environmental Studies Animal Care and Use Committee.

\section{Study protocol}

Mice were divided into eight groups (fig. 1). The vehicle group received PBS at pH 7.4 (Nissui Pharmaceutical Co., Tokyo, Japan) containing $0.05 \%$ Tween 80 (Nacalai Tesque, Kyoto, Japan) once a week for 6 weeks. The ovalbumin (OVA) group received $1 \mu \mathrm{g}$ of OVA (Sigma Chemical, St. Louis, MO, USA) dissolved in the same vehicle bi-weekly for 6 weeks. The NQ group received $\mathrm{NQ}$ at a dose of $1.58 \mathrm{ng} \cdot \mathrm{animal}^{-1}$, $15.8 \mathrm{ng} \cdot$ animal $^{-1}$ or $158 \mathrm{ng} \cdot$ animal $^{-1}$ (NQ $(1.58 \mathrm{ng})$, NQ (15.8 ng) and NQ (158 ng) groups) dissolved in the same vehicle every week for 6 weeks. The NQ and OVA group received the combined treatment in the same protocol as the NQ and the OVA groups. For each group, vehicle, NQ, OVA, or OVA and NQ was dissolved in $0.1-\mathrm{mL}$ aliquots and inoculated by the intratracheal route through a polyethylene tube under anaesthesia with $4 \%$ halothane (Hoechst Japan Ltd, Tokyo, Japan) as described previously [8, 27]. The animals were studied $24 \mathrm{~h}$ after the last intratracheal administration.

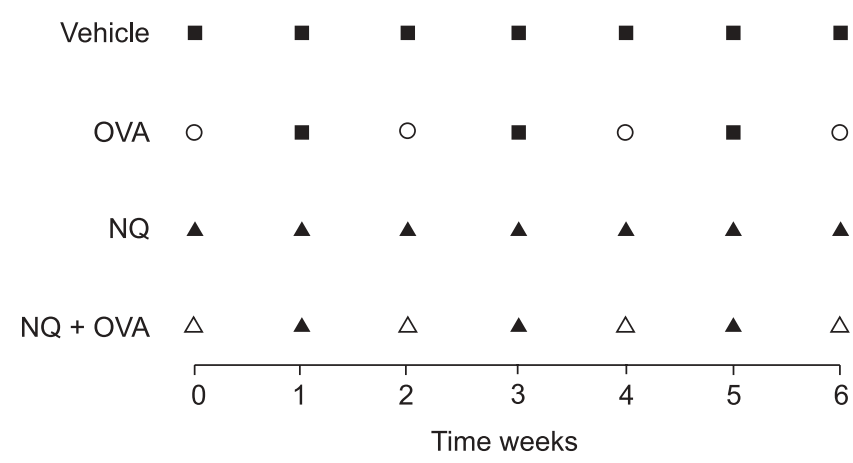

FIGURE 1. Experimental design. $\mathbf{a}$ : Vehicle only; $\bigcirc$ : ovalbumin (OVA; $1 \mu \mathrm{g} \cdot$ animal $^{-1}$ ); $\mathbf{\Delta}$ : naphthoquinone (NQ; $1.58,15.8$ or $158 \mathrm{ng} \cdot$ animal $\left.^{-1}\right) ; \triangle: N Q$ plus OVA.

\section{Blood retrieval and analysis}

Mice were anaesthetised with diethylether. The chest and abdominal walls were opened, and blood was retrieved by cardiac puncture. Plasma was prepared and frozen at $-80^{\circ} \mathrm{C}$ until assayed for antigen-specific $\operatorname{IgG}_{1}$ and $\operatorname{IgG}_{2 \mathrm{a}}$.

\section{Bronchoalveolar lavage}

The trachea was cannulated after the collection of blood. The lungs were lavaged with $1.2 \mathrm{~mL}$ of sterile saline at $37^{\circ} \mathrm{C}$, instilled bilaterally by syringe. The bronchoalveolar lavage fluid (BALF) was harvested by gentle aspiration. This procedure was conducted a further two times. The average volume retrieved was $90 \%$ of the $3.6 \mathrm{~mL}$ that was instilled; the amounts did not differ by treatment. The fluid collections were combined and cooled to $4^{\circ} \mathrm{C}$. The BALF was centrifuged at $300 \times g$ for $10 \mathrm{~min}$, and the total cell count was determined on a fresh fluid specimen using a haemocytometer. Differential cell counts were assessed on cytological preparations. Slides were prepared using Autosmear (Sakura Seiki Co., Tokyo, Japan) and were stained with Diff-Quik (International Reagents Co., Kobe, Japan). A total of 500 cells were counted under oil immersion microscopy ( $n=7-8$ in each group).

\section{Histological evaluation of leukocyte accumulation and goblet cell metaplasia in the lung}

After the collection of blood, the lungs were fixed by intratracheal instillation of $10 \%$ neutral phosphate-buffered formalin at a pressure of $20 \mathrm{cmH}_{2} \mathrm{O}$. After separation of the lobe, 2-mm thick blocks were taken for paraffin embedding. Sections $3 \mu \mathrm{m}$ thick were stained with haematoxylin and eosin stain (HE) to observe and evaluate the degree of infiltration of eosinophils or neutrophils and mononuclear cells around the airways. The sections were stained with periodic acid Schiff (PAS) to evaluate the degree of proliferation of goblet cells in the bronchial epithelium. Histological analyses were performed using a microscope. The degree of eosinophil or neutrophil and mononuclear cell infiltration around the airways and the proliferation of goblet cells in the bronchial epithelium were graded in a blind fashion: 0 : not present; 1 : very slight; 2 : slight; 3 : moderate; 4 : moderate to marked; 5 : marked. An inflammatory reaction affecting $<20 \%$ of the airways was defined as " 1 " ( $<20 \%$ goblet cells stained with PAS), " 2 " was defined as $20-40 \%$ of the airways were affected, " 3 " $40-60 \%$, " 4 " $60-80 \%$ and " 5 ": $>80 \%$ of the airways were affected ( $n=4-5$ in each group) [10].

\section{Quantification of cytokine and chemokine protein levels in the lung}

In a separate series of experiments, the animals were exsanguinated and the lungs were subsequently homogenised with $10 \mathrm{mM}$ potassium phosphate buffer ( $\mathrm{pH}$ 7.4) containing $0.1 \mathrm{mM}$ ethylenediaminetetraacetic acid (Sigma), $0.1 \mathrm{mM}$ phenylmethanesulphonyl fluoride (Nacalai Tesque), $1 \mu \mathrm{M}$ pepstatin A (Peptide Institute, Osaka, Japan) and $2 \mu \mathrm{M}$ leupeptin (Peptide Institute) as described previously [8]. The homogenates were then centrifuged at $105,000 \times g$ for $1 \mathrm{~h}$. The supernatants were stored at $-80^{\circ} \mathrm{C}$. ELISAs for IL-4 (Amersham, Buckinghamshire, UK), IL-5 (Endogen, Cambridge, MA, USA), interferon (IFN)- $\gamma$ (R\&D systems, Minneapolis, MN, USA), eotaxin (R\&D systems), MCP-1 (R\&D systems) and $K C$ (R\&D systems) in the lung tissue 
supernatants were conducted using matching antibody pairs according to the manufacturers' instructions. The second antibodies were conjugated to horseradish peroxidase. Subtractive readings of $550 \mathrm{~nm}$ from the readings at $450 \mathrm{~nm}$ were converted to $\mathrm{pg} \cdot \mathrm{mL}^{-1}$ using values obtained from standard curves generated with the limits of detection of $5 \mathrm{pg} \cdot \mathrm{mL}^{-1}, 5 \mathrm{pg} \cdot \mathrm{mL}^{-1}, 5 \mathrm{pg} \cdot \mathrm{mL}^{-1}, 3 \mathrm{pg} \cdot \mathrm{mL}^{-1}, 1.5 \mathrm{pg} \cdot \mathrm{mL}^{-1}$ and $2 \mathrm{pg} \cdot \mathrm{mL}^{-1}$ for IL-4, IL-5, IFN- $\gamma$, eotaxin, MCP-1 and KC, respectively $(n=7-8$ in each group).

\section{Antigen-specific IgG determination}

Antigen-specific $\operatorname{IgG}_{1}$ or $\operatorname{IgG}_{2 a}$ antibodies were measured by ELISA with solid-phase antigen $[8,10]$. In brief, microplate wells (Dynatech, Chantilly, VA, USA) were coated with OVA overnight at $4{ }^{\circ} \mathrm{C}$ and then incubated at room temperature for $1 \mathrm{~h}$ with PBS containing 1\% bovine serum albumin (Sigma) containing $0.01 \%$ thimerosal (Nakalai Tesque). After washing, diluted samples were introduced to the microplate and incubated at room temperature for $1 \mathrm{~h}$. After another washing, the wells were incubated at room temperature for $1 \mathrm{~h}$ with biotinylated rabbit anti-mouse $\mathrm{IgG}_{1}$ or $\mathrm{IgG}_{2 \mathrm{a}}$ (Zymed Laboratories, San Francisco, CA, USA). After another washing, the wells were incubated with horseradish-peroxidase-conjugated streptavidin (Sigma) at room temperature for $1 \mathrm{~h}$. The wells were then washed and incubated with o-phenylenediamine and hydrogen peroxide in the dark at room temperature for $30 \mathrm{~min}$. The enzyme reaction was stopped with $4 \mathrm{~N}$ sulphuric acid. Absorbance was read at $492 \mathrm{~nm}$. Each plate incubated a previously screened standard plasma that contained a high titre of anti-OVA antibodies. The results were expressed in titres, calculated based on the titres of the standard plasma. Cut-off values for antibody-positive plasma were set to hold as the mean value of absorbance of pre-immune plasma $(n=7-8$ in each group).

\section{Statistical analysis}

Data were reported as mean \pm SEM. Differences among groups were analysed by ANOVA followed by Fisher's PLSD test. Significance was assigned to $\mathrm{p}$-values $<0.05$.

\section{RESULTS}

\section{$N Q$ accelerates antigen-related airway inflammation}

To evaluate the effects of NQ on allergic airway inflammation, the cellular profile of BALF and lung histology in eight groups of mice was investigated $24 \mathrm{~h}$ after the last intratracheal instillation.

There was a tendency that the number of neutrophils in BALF was greater in the NQ or the OVA group than in the vehicle group (fig. 2a). The number was greater in the NQ and OVA groups than in the OVA $(\mathrm{p}<0.05$ versus the NQ (158 ng)+OVA group) or NQ ( $<<0.01$ versus the NQ (158 ng) and OVA group) group. The number of macrophages was greater in the NQ groups than in the vehicle group ( $<<0.05$ for the NQ (15.8 ng) group; fig. $2 b$ ). There was a tendency that the number of eosinophils was greater in the OVA group than in the vehicle group (fig. 2c). The number was significantly greater in the NQ $(158 \mathrm{ng})$ and OVA group than in the OVA $(\mathrm{p}<0.01)$ or the NQ $(\mathrm{p}<0.05)$ group. There was a tendency that the number of mononuclear cells was greater in the NQ (15.8 ng) or the OVA group than in the vehicle group (fig. $2 \mathrm{~d}$ ). The number was further greater in the NQ and OVA groups than in the OVA group ( $\mathrm{p}<0.05$ versus the NQ (158 ng) and OVA group) or in the NQ groups (NS).

To quantify the infiltration of inflammatory cells around the airways, the magnitude of the histopathological changes in the lung specimens stained with $\mathrm{HE}$ was estimated (fig. 3). Instillation of $15.8 \mathrm{ng}$ of NQ increased the number of infiltrated eosinophils in comparison with vehicle instillation. Instillation of OVA increased the numbers in comparison with vehicle instillation (fig. 3a and fig. 4). Combined treatment with NQ and OVA significantly increased the number in comparison with OVA treatment alone ( $\mathrm{p}<0.05$ versus the NQ (1.58 $\mathrm{ng})$ and OVA group, $\mathrm{p}<0.01$ versus the NQ (15.8 ng) and OVA group or the NQ (158 ng) and OVA group) or NQ treatment alone $(\mathrm{p}<0.05$ versus the NQ (1.58 ng) and OVA group, $\mathrm{p}<0.01$ versus
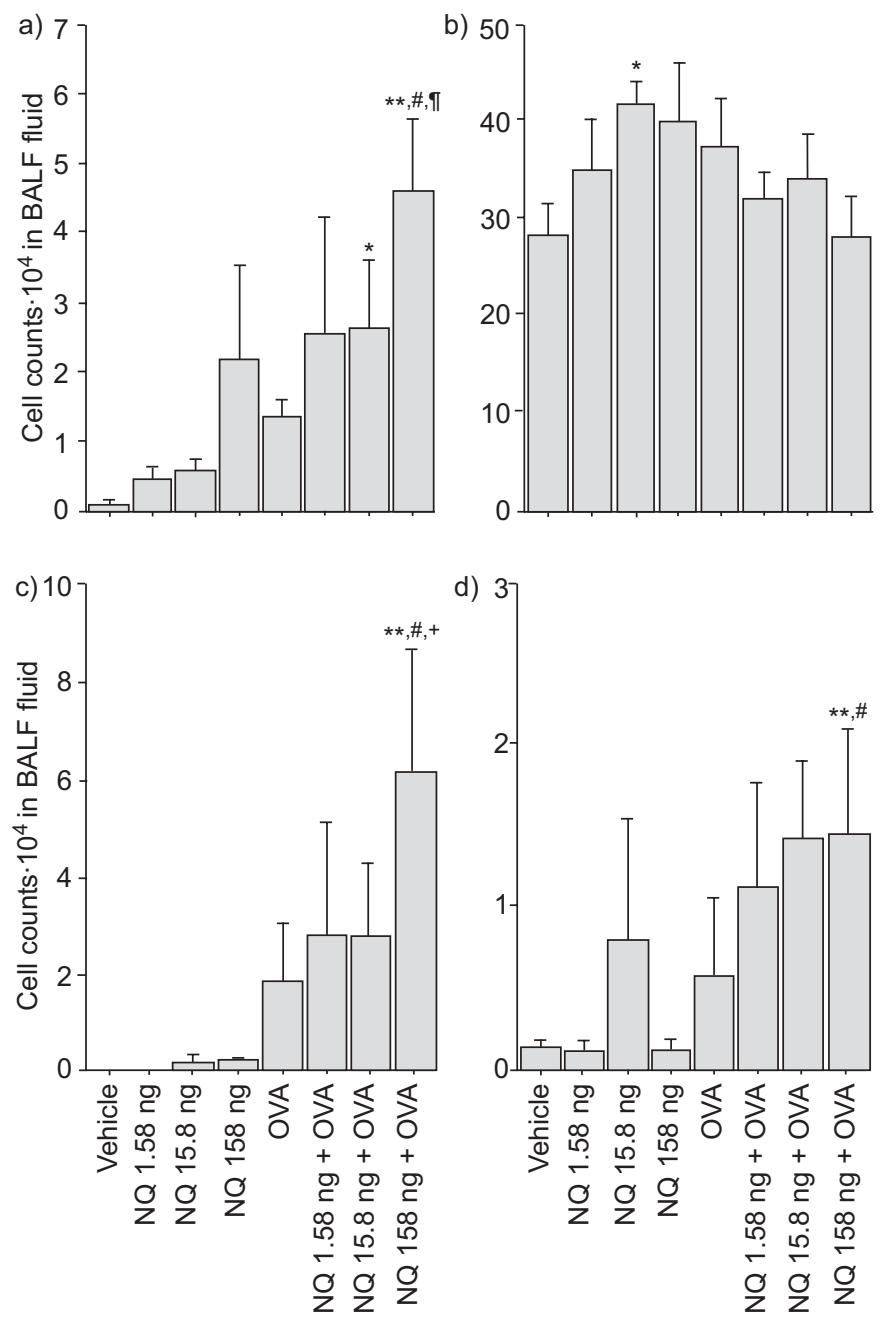

FIGURE 2. Cellular profile of a) neutrophils, b) macrophages, c) eosinophils and d) mononuclear cells in bronchoalveolar lavage fluid (BALF). Eight groups of mice were intratracheally administered vehicle, naphthoquinone (NQ; $1.58,15.8$ or $158 \mathrm{ng} \cdot$ animal $^{-1}$ ), ovalbumin (OVA), or a combination of NQ and OVA for 6 weeks. Bronchoalveolar lavage was conducted $24 \mathrm{~h}$ after the last intratracheal instillation. Differential cell counts were assessed with Diff-Quik staining. Results are presented as mean \pm SEM $\left(n=7-8\right.$ in each group). ${ }^{*}: p<0.05$ versus vehicle; ${ }^{*}: p<0.01$ versus vehicle; ${ }^{\#}: p<0.05$ versus OVA; ${ }^{\uparrow}: p<0.01$ versus $N Q ;{ }^{+}: p<0.01$ versus OVA; ${ }^{\S}$ : $\mathrm{p}<0.06$ versus $N Q$ 

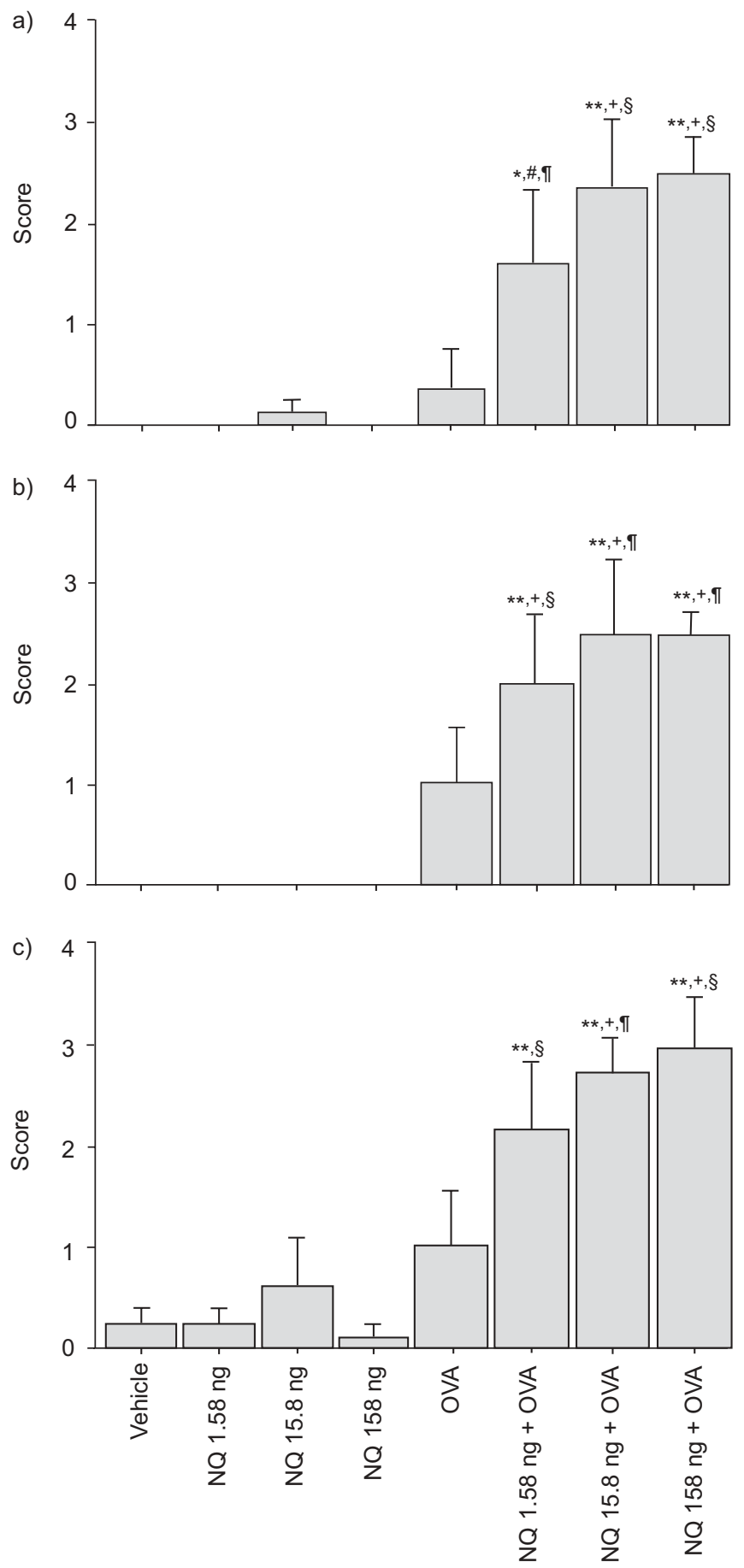

FIGURE 3. Degree of infiltration of inflammatory cells around the airways and goblet cells in the bronchial epithelium. Animals received intratracheal instillation of vehicle, naphthoquinone (NQ; $1.58,15.8$ or $158 \mathrm{ng} \cdot$ animal $^{-1}$ ), ovalbumin (OVA) or OVA and NQ for 6 weeks. Lungs were removed and fixed $24 \mathrm{~h}$ after the last intratracheal administration. Sections were stained with haematoxylin and eosin for measurement of inflammatory cells around the airways or with periodic acid Schiff for goblet cells in the bronchial epithelium. Degree of infiltration of a) eosinophils, b) neutrophils and mononuclear cells and c) goblet cells was estimated. Values are mean \pm SEM ( $n=4-5$ in each group). *: $p<0.05$ versus vehicle; ${ }^{* *}: p<0.01$ versus vehicle; ${ }^{\#}: p<0.05$ versus NQ; ${ }^{\natural}: p<0.05$ versus $\mathrm{OVA}^{+}{ }^{+}: p<0.01$ versus NQ; ${ }^{\varsigma}$ : $p<0.01$ versus OVA. the NQ (15.8 ng) and OVA group or the NQ (158 ng) and OVA group). Instillation of NQ showed negligible increases in the numbers of infiltrated neutrophils and mononuclear cells as compared with vehicle instillation. There was a tendency that instillation of OVA increased the numbers as compared with vehicle instillation (fig. $3 \mathrm{~b}$ and fig. 4). The NQ plus OVA groups showed significant increases in the number as compared with the OVA group $(\mathrm{p}<0.01$ versus the NQ $(1.58 \mathrm{ng})$ and OVA group, the NQ (15.8 ng) and OVA group or the NQ (158 ng) and OVA group) or the NQ groups ( $\mathrm{p}<0.01$ versus the NQ (1.58 ng) and OVA group, the NQ (15.8 $\mathrm{ng})$ and OVA group or the NQ (158 ng) and OVA group).

\section{Effects of NQ on the goblet cell metaplasia in the airways after antigen challenge}

To evaluate bronchial epithelial injury and hypersecretion of mucus, lung sections were stained with PAS. There was a tendency that the number of goblet cells was slightly greater in the NQ $(15.8 \mathrm{ng})$ group or in the OVA group than in the vehicle group (fig. $3 \mathrm{c}$ and fig. 5). The combined treatment with NQ and OVA significantly increased the number in comparison with the OVA $(\mathrm{p}<0.01)$ or the NQ treatment $(\mathrm{p}<0.05$ versus the NQ (15.8 ng) and OVA group, $\mathrm{p}<0.01$ versus the NQ (158 ng) and OVA group).

\section{Effects of NQ on local expression of cytokines in the presence of antigen}

To explore the role of local expression of T-helper (Th)2 cytokines, such as IL-4 and IL-5, or that of a Th1 cytokine, IFN$\gamma$, in the enhancing effects of NQ on antigen-related airway inflammation, the protein levels in the lung tissue supernatants were quantified $24 \mathrm{~h}$ after the last intratracheal instillation (table 1). The protein level of IL-4 was significantly greater in the NQ (158 ng; $\mathrm{p}<0.05)$, the OVA and the NQ (158 ng) and OVA $(p<0.01)$ groups than in the vehicle group. The level was greater in the NQ (158 ng) and OVA group than in the NQ $(158$ ng; $\mathrm{p}<0.05)$. However, the level was not significantly different between the OVA and the NQ (158 ng) and OVA groups. Protein levels of IL-5 and IFN- $\gamma$ in the NQ (158 ng) group were comparable to those in the vehicle group. There was a tendency that OVA exposure increased the levels of IL-5 in comparison with vehicle exposure. The level of IL-5 was greater in the NQ (158 $\mathrm{ng})$ and OVA group than in the NQ $(158 \mathrm{ng})$ group $(\mathrm{p}<0.01)$. The IFN- $\gamma$ level was greater in the NQ (158 ng) and OVA group than in the vehicle (NS), the NQ (NS) or the OVA $(\mathrm{p}<0.05)$ group.

\section{Effects of NQ on local expression of chemokines in the presence of antigen}

To explore the role of local expression of chemokines such as eotaxin, MCP-1, and KC in the enhancing effects of NQ on antigen-related airway inflammation, the protein levels of the chemokines in the lung tissue supernatants were quantitfied $24 \mathrm{~h}$ after the last intratracheal instillation (table 2). These protein levels in the NQ (158 ng) group were comparable to those in the vehicle group. OVA exposure increased the levels of the chemokines as compared with vehicle exposure $(p<0.05$ for MCP-1). The level of eotaxin was greater in the NQ (158 ng) and OVA group than in the NQ $(158 \mathrm{ng} ; \mathrm{p}<0.05)$ or the OVA group (NS). The levels of MCP-1 and KC were greater in the 

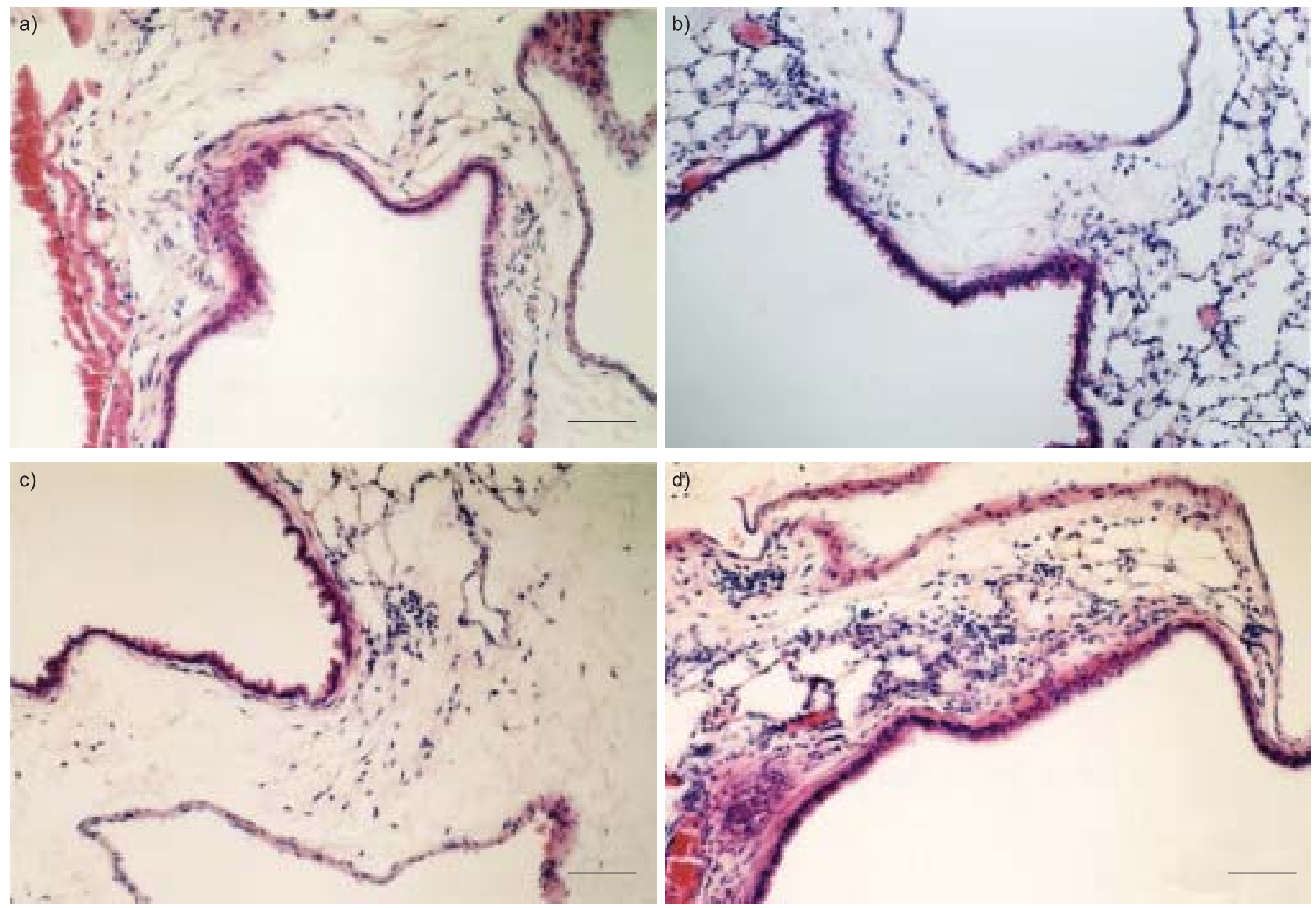

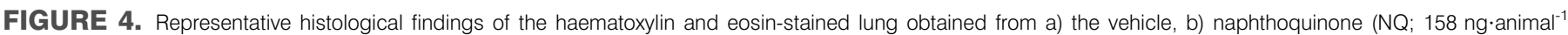

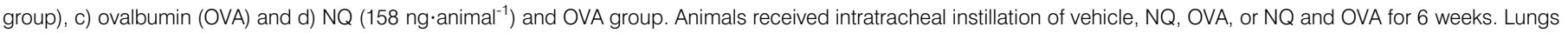
were removed and fixed $24 \mathrm{~h}$ after the last intratracheal administration. Scale bars $=100 \mu \mathrm{m}$.

NQ (158 ng) and OVA group than in the NQ (158 ng; $\mathrm{p}<0.01$ for MCP-1; $<<0.05$ for $K C)$ group or the OVA $(\mathrm{p}<0.05)$ group.

\section{NQ has adjuvant activity for antigen-specific production of Ig $G_{1}$ and $/ g G_{2 a}$}

To examine whether NQ has adjuvant activity for antigenspecific Ig production, antigen-specific $\operatorname{IgG}_{1}$ (fig. 6) and $\operatorname{IgG}_{2 a}$ (fig. 7) was measured $24 \mathrm{~h}$ after the last intratracheal instillation. There was a tendency that the titre of antigen-specific $\mathrm{IgG}_{1}$ was greater in the OVA group than in the vehicle group. The combination of NQ and OVA, in particular at a dose of $1.58 \mathrm{ng} \cdot$ animal $^{-1}$, increased antigen-specific production of $\mathrm{IgG}_{1}$ as compared with OVA alone $(\mathrm{p}<0.05$ for NQ $(1.58 \mathrm{ng})$ and OVA). There was a tendency that the titre of antigen-specific $\mathrm{IgG}_{2 \mathrm{a}}$ was greater in the OVA group than in the vehicle group. The combination of NQ plus OVA, in particular at a dose of $158 \mathrm{ng} \cdot$ animal $^{-1}$, increased antigen-specific production of $\operatorname{IgG}_{2 a}$ as compared with vehicle, NQ, or OVA alone $(\mathrm{p}<0.05$ for $\mathrm{NQ}$ (158 ng) and OVA).

\section{DISCUSSION}

The present study has demonstrated that NQ administered by the intratracheal route deteriorates antigen-related airway inflammation in mice, which is characterised by the infiltration of inflammatory leukocytes in both the bronchoalveolar spaces and the lung parenchyma. NQ also exaggerates antigen-related goblet cell metaplasia. The enhancing effects are concomitant with the increased lung expression of IL-5, eotaxin and especially with that of MCP-1 and KC. In addition, NQ exhibits adjuvant activity for the antigen-specific production of $\operatorname{IgG}_{1}$ and $\operatorname{IgG}_{2 \mathrm{a}}$.

The current authors have previously shown that intratracheal administration of DEP enhances airway inflammation related to antigen [8]. DEP consist of carbonaceous nuclei and a vast number of organic chemical compounds such as polyaromatic hydrocarbons, aliphatic hydrocarbons, heterocycles and quinones [26, 28, 29]. Previous in vitro studies have indicated that exposure of macrophages to organic chemicals extracted from DEP increases the oxidative stress-inducible protein haeme oxygenase-1 [12] and results in induction of apoptosis [11]. Organic chemicals in DEP can also affect inflammatory effector leukocytes [13-15] and trigger the release of pro-inflammatory molecules such as IL-1, IL- 8 , tumour necrosis factor- $\alpha$ and RANTES (regulated on activation normal T-cells expressed and secreted) in vitro $[15,16]$. The current authors have also recently shown that intratracheal instillation of organic chemicals from 

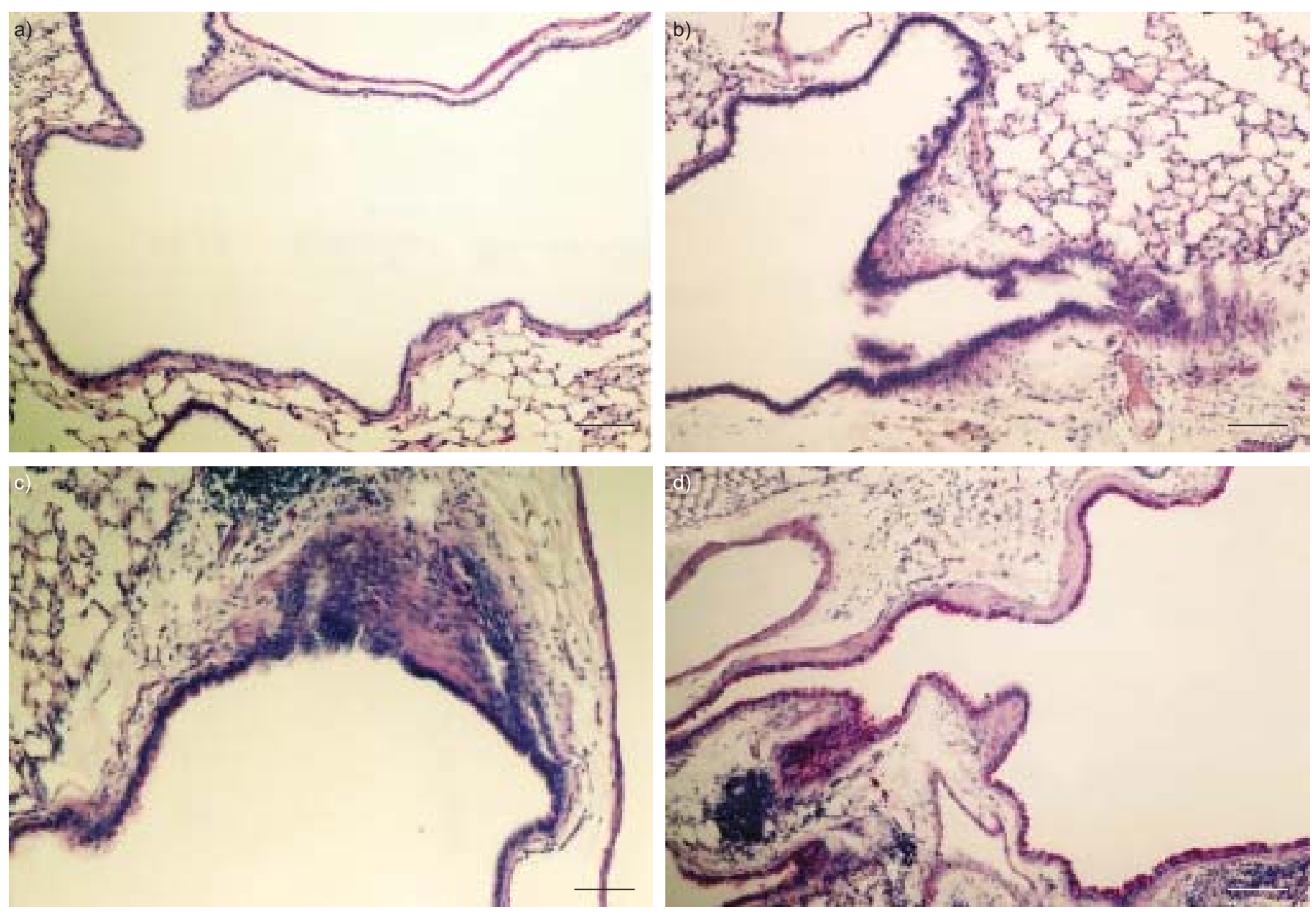

FIGURE 5. Representative histological findings of the periodic acid Schiff (PAS)-stained lung obtained from a) the vehicle, b) naphthoquinone (NQ; $158 \mathrm{ng} \cdot \mathrm{animal}^{-1}$ group), c) ovalbumin (OVA) and d) NQ (158 ng.animal ${ }^{-1}$ ) and OVA group. Animals received intratracheal instillation of vehicle, NQ, OVA, or NQ and OVA for 6 weeks. Lungs were removed and fixed $24 \mathrm{~h}$ after the last intratracheal administration. Scale bars $=100 \mu \mathrm{m}$.

DEP enhances the neutrophilic lung inflammation related to endotoxin in vivo [27]. Furthermore, a more recent study has shown that organic chemicals in DEP, rather than their carbonaceous nuclei, predominantly enhance antigen-related airway inflammation in mice [17]. However, the responsible organic chemicals in DEP have not been fully identified.
Quinones are present in DEP $[18,19]$ and possess toxicological properties to serve as alkylating agents and to interact with, for example, flavoproteins to generate ROS, which can induce biological injury [20-23]. It has recently been shown, again by the present authors, that single intratracheal administration of $P Q$, one of the quinones present in DEP $[19,23]$, can induce

TABLE 1 Protein levels of cytokines in the lung

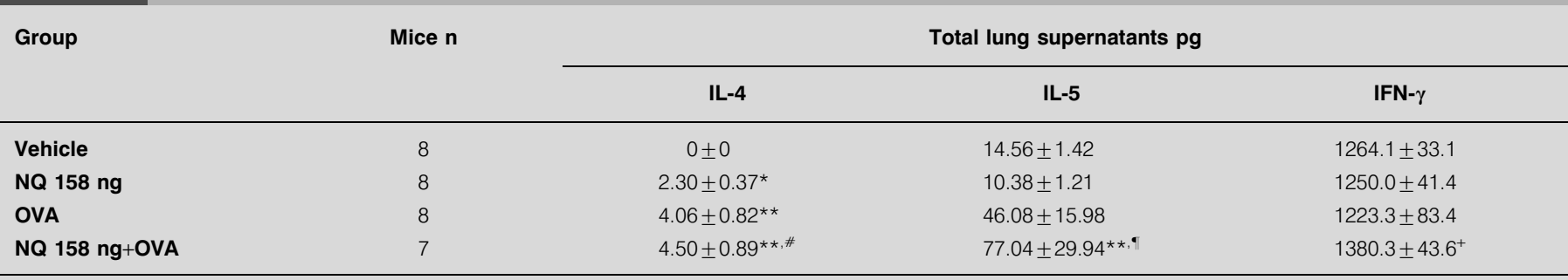

Data are presented as mean \pm SEM, unless otherwise stated. Four groups of mice were intratracheally inoculated with vehicle, naphthoquinone (NQ; $158 \mathrm{ng} \cdot$ animal $^{-1}$ ), ovalbumin (OVA), or the combination of NQ and OVA for 6 weeks. Lungs were removed and frozen $24 \mathrm{~h}$ after the last intratracheal administration. Protein levels in the lung tissue supernatants were analysed using ELISA. *: $p<0.05$ versus vehicle; **: $p<0.01$ versus vehicle; ${ }^{*}: p<0.05$ versus NQ (158 ng). ": $p<0.01$ versus NQ (158 ng). ${ }^{+}$: $\mathrm{p}<0.05$ versus OVA. 
TABLE 2 Protein levels of chemokines in the lung.

\begin{tabular}{|c|c|c|c|c|}
\hline \multirow[t]{2}{*}{ Group } & \multirow[t]{2}{*}{ Mice $\mathbf{n}$} & \multicolumn{3}{|c|}{ Total lung supernatants pg } \\
\hline & & Eotaxin & MCP-1 & KC \\
\hline Vehicle & 8 & $66.31 \pm 2.52$ & $18.44 \pm 4.05$ & $17.90 \pm 4.61$ \\
\hline NQ $158 \mathrm{ng}$ & 8 & $74.76 \pm 3.50$ & $21.14 \pm 3.72$ & $19.81 \pm 6.74$ \\
\hline OVA & 8 & $233.70 \pm 90.78$ & $44.64 \pm 9.37^{\star}$ & $32.38 \pm 10.46$ \\
\hline
\end{tabular}

recruitment of inflammatory cells, such as eosinophils and neutrophils, with the local expression of pro-inflammatory molecules such as IL-5 and eotaxin in vivo [24]. The study demonstrated that pulmonary exposure to PQ enhances antigen-related airway inflammation in vivo [25]. Conversely, $\mathrm{NQ}$, another extractable chemical compound in DEP, generates free radicals, binds to thiol-containing proteins and irreversibly inactivates them [26]. In the present study, the numbers of eosinophils, neutrophils, and mononuclear cells in the BALF and in the lung tissues were greater in the NQ plus OVA groups than in the OVA group in a dose-dependent manner with overall trend. The results indicate that NQ can exacerbate antigen-related airway inflammation. Furthermore, in a previous study [25], the current authors have shown that PQ has

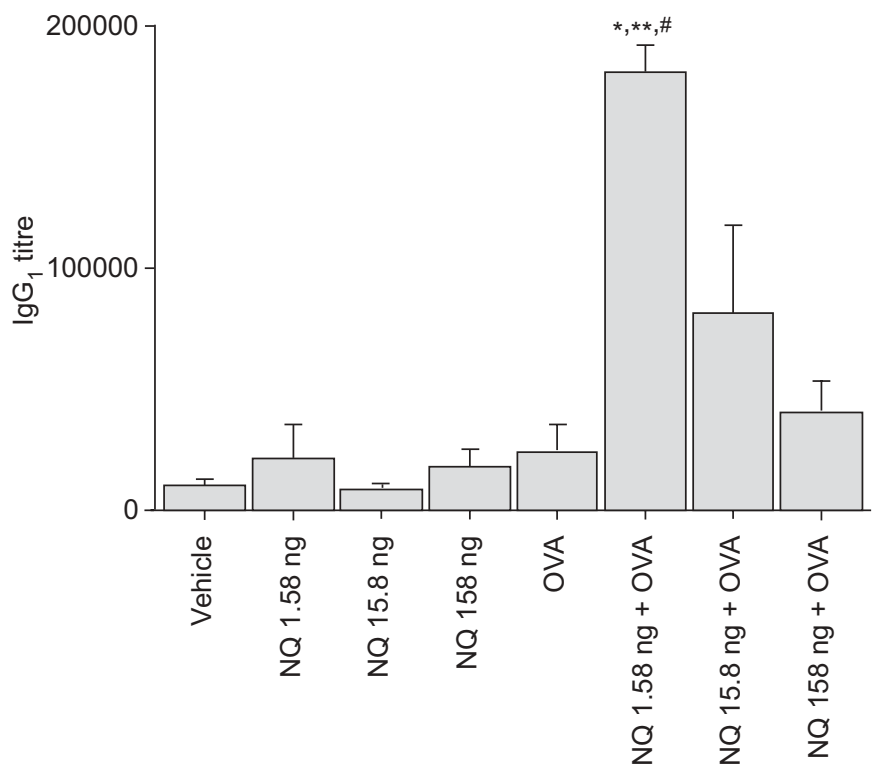

FIGURE 6. Antigen-specific immunoglobulin (lg) $\mathrm{G}_{1}$ titres. Eight groups of mice were intratracheally administered vehicle, ovalbumin (OVA), naphthoquinone (NQ;

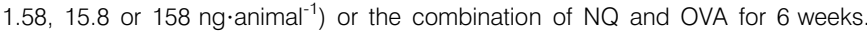
Plasma samples were retrieved $24 \mathrm{~h}$ after the last intratracheal instillation. Antigenspecific $\lg G_{1}$ was analysed using ELISA. Results are expressed as mean \pm SEM $\left(n=7-8\right.$ in each group). ${ }^{* *}: p<0.01$ versus vehicle; ${ }^{*}: p<0.05$ versus OVA; ${ }^{*}$ : $p<0.01$ versus $N Q$ not shown significant aggravating effects on the magnitude of airway inflammation and goblet cell metaplasia in the lung histology. In the present study, combined treatment with NQ and antigen histologically exacerbated airway inflammation and goblet cell metaplasia as compared with treatment with antigen alone. The previous study [25] and the present results suggest that $N Q$, rather than $P Q$, may be an important quinone involved in the enhancing effects of DEP on antigen-related airway inflammation.

Allergic asthma is often associated with predominant local expression of Th2 type cytokines including IL-4 and IL-5. Among chemokines, eotaxin is essential for eosinophil recruitment in antigen-related airway inflammation [30, 31]. In fact,

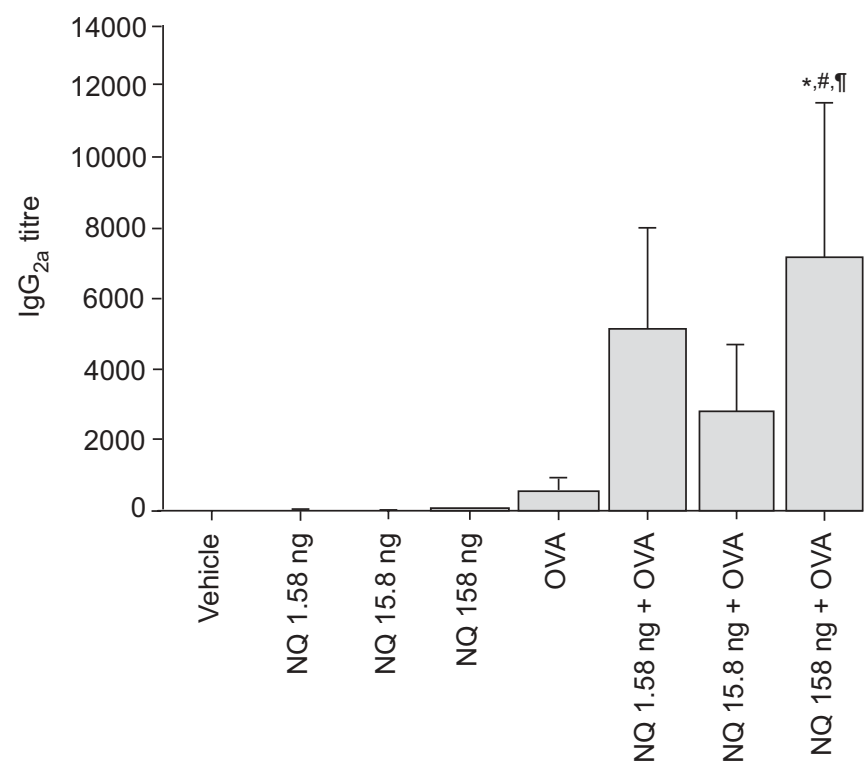

FIGURE 7. Antigen-specific immunoglobulin ( $(\mathrm{g}) \mathrm{G}_{2 a}$ titres. Eight groups of mice were intratracheally administered vehicle, ovalbumin (OVA), naphthoquinone (NQ; $1.58,15.8$ or $158 \mathrm{ng} \cdot$ animal $^{-1}$ ) or the combination of NQ and OVA for 6 weeks. Plasma samples were retrieved $24 \mathrm{~h}$ after the last intratracheal instillation. Antigenspecific $\lg G_{2 a}$ was analysed using ELISA. Results are expressed as mean \pm SEM ( $n=7-8$ in each group). ${ }^{*}: p<0.05$ versus vehicle; ${ }^{*}: p<0.05$ versus OVA; ${ }^{n}: p<0.05$ versus $N Q$ 
the previous studies have confirmed that the exaggerated allergic airway inflammation induced by DEP parallels the local elevation of the inflammatory protein levels [7, 8]. MCP-1 has a chemoattractant effect on CD4+ and CD8+ T-lymphocytes [32] and also plays a role in recruitment of eosinophils to inflammatory sites [33]. KC induces airway inflammation with mucus hypersecretion [34-36] and also plays a role in airway hyperresponsiveness [36]. Therefore, local expression of the cytokines and chemokines were measured in the present study. NQ further enhanced the lung expression of these proteins, especially those of MCP-1 and KC, which were elevated by the antigen challenge alone. Thus, the results suggest that NQ aggravates antigen-related airway inflammation, at least in part, via the enhancement of the local expression of these proteins. Interestingly, in the previous study, PQ did not significantly enhance the expression of these proteins in the presence of antigen ([25] and unpublished observations). The results may also indicate that the enhancing effects of NQ on antigen-related airway inflammation may be stronger than those of PQ. It is also possible that the enhancing effects of NQ are mediated through different pathways from those of PQ. Future studies are needed to clarify the mechanisms of deteriorated airway inflammation induced by the quinones.

IgG with antigen is a strong agonist for eosinophil degradation in vitro [37]. Furthermore, late asthmatic reactions are associated with IgG antibody [38]. The current authors have reported that DEP enhance antigen-specific production of IgG induced by OVA in vivo $[7,8]$. In the present study, the combined intratracheal administration of $\mathrm{NQ}$ and antigen induced a greater increase in both OVA-specific $\operatorname{IgG}\left(\operatorname{IgG}_{1}\right.$ and $\operatorname{IgG}_{2 a}$ titres) than the administration of vehicle, NQ or antigen alone. It is likely that NQ also plays a role in the enhancing effects of DEP in view of antigen-specific Ig production. The magnitude of inflammatory response after treatment with NQ appeared to be directly correlated with dose, with the highest response at $158 \mathrm{ng}$. However, this was not the case for OVA-specific $\operatorname{IgG}_{1}$. A bell-shaped dose response was observed with the highest immune response at a much lower dose $(1.58 \mathrm{ng})$ followed by a decline with increasing doses. The 100-fold difference in optimal dose might be explained by a shift towards other IgGisotypes at higher doses. Indeed, OVA-specific $\operatorname{IgG}_{2 a}$ titre, a hallmark of Th1-biased immune response, was highest in the NQ (158 ng) and OVA group, although this titre did not exhibit an apparent dose-response curve. In addition, there was a tendency that IFN- $\gamma$, representative of Th1 cytokine, was higher in the NQ (158 ng) and OVA group than in the OVA group. These results indicate that $\mathrm{NQ}$ enhances Th2-related $\mathrm{IgG}_{1}$ production at a lower dose, whereas it increases Th1-related $\mathrm{IgG}_{2 a}$ production at higher doses. Conversely, titres of $\mathrm{IgE}$, another critical Ig in the hallmark of asthma, were not significantly different between the experimental groups (data not shown). Significant production of antigen-specific IgE in the antigen-sensitised and challenged group as compared with the vehicle group has been found at 9 weeks or later in previous experimental protocol [8]. Thus, another experimental protocol may be needed in future to elucidate the effects of NQ on adjuvant activity for antigen-specific $\operatorname{IgE}$ production. Collectively, the detailed impacts of NQ on humoral responses and their mechanisms await further studies.
In addition to this, there is a correlation between airway inflammation and airway responsiveness [39]. Furthermore, goblet cell metaplasia is strongly related to the airway pathophysiology [40]. In the present study, it was observed that NQ enhanced airway inflammation with goblet cell metaplasia was related to repetitive antigen exposure, suggesting that NQ may facilitate airway hyperresponsiveness of the condition. The current authors are conducting further experiments to study the effects of NQ on airway hyperrreactivity.

In conclusion, it has been shown that naphthoquinone can enhance allergic airway inflammation. The enhancing effects are concomitant with the increased lung expression of interleukin-4, interleukin-5, eotaxin, and especially with that of macrophage chemoattractant protein-1 and keratinocyte chemoattractant. These results suggest that environmental quinones are implicated in the increasing prevalence of allergic asthma in recent years and may play, at least in part, a role in the toxicity of diesel exhaust particles against subjects with allergic diseases.

\section{REFERENCES}

1 McClellan RO. Health effects of exposure to diesel exhaust particles. Annu Rev Pharmacol Toxicol 1987; 27: 279-300.

2 Ichinose T, Furuyama A, Sagai M. Biological effects of diesel exhaust particles (DEP). II. Acute toxicity of DEP introduced into lung by intratracheal instillation. Toxicology 1995; 99: 153-167.

3 Nikasinovic L, Momas I, Just J. A review of experimental studies on diesel exhaust particles and nasal epithelium alterations. J Toxicol Environ Health B Crit Rev 2004; 7: 81-104.

4 Takizawa H. Diesel exhaust particles and their effect on induced cytokine expression in human bronchial epithelial cells. Curr Opin Allergy Clin Immunol 2004; 4: 355-359.

5 Muranaka M, Suzuki S, Koizumi K, et al. Adjuvant activity of diesel-exhaust particulates for the production of IgE antibody in mice. J Allergy Clin Immunol 1986; 77: 616-623.

6 Takafuji S, Suzuki S, Koizumi K, et al. Diesel-exhaust particulates inoculated by the intranasal route have an adjuvant activity for IgE production in mice. J Allergy Clin Immunol 1987; 79: 639-645.

7 Miyabara Y, Yanagisawa R, Shimojo N, et al. Murine strain differences in airway inflammation caused by diesel exhaust particles. Eur Respir J 1998; 11: 291-298.

8 Takano H, Yoshikawa T, Ichinose T, Miyabara Y, Imaoka K, Sagai M. Diesel exhaust particles enhance antigen-induced airway inflammation and local cytokine expression in mice. Am J Respir Crit Care Med 1997; 156: 36-42.

9 Takano H, Ichinose T, Miyabara Y, Yoshikawa T, Sagai M. Diesel exhaust particles enhance airway responsiveness following allergen exposure in mice. Immunopharmacol Immunotoxicol 1998; 20: 329-336.

10 Ichinose T, Takano H, Miyabara Y, Sagai M. Long-term exposure to diesel exhaust enhances antigen-induced eosinophilic inflammation and epithelial damage in the murine airway. Toxicol Sci 1998; 44: 70-79.

11 Hiura TS, Kaszubowski MP, Li N, Nel AE. Chemicals in diesel exhaust particles generate reactive oxygen radicals 
and induce apoptosis in macrophages. J Immunol 1999; 163: 5582-5591.

12 Li N, Venkatesan MI, Miguel A, et al. Induction of heme oxygenase-1 expression in macrophages by diesel exhaust particle chemicals and quinones via the antioxidantresponsive element. I Immunol 2000; 165: 3393-3401.

13 Kanemitsu H, Nagasawa S, Sagai M, Mori Y. Complement activation by diesel exhaust particles (DEP). Biol Pharm Bull 1998; 21: 129-132.

14 Terada N, Maesako K, Hiruma K, et al. Diesel exhaust particulates enhance eosinophil adhesion to nasal epithelial cells and cause degranulation. Int Arch Allergy Immunol 1997; 114: 167-174.

15 Yang HM, Ma JY, Castranova V, Ma JK. Effects of diesel exhaust particles on the release of interleukin-1 and tumor necrosis factor- $\alpha$ from rat alveolar macrophages. Exp Lung Res 1997; 23: 269-284.

16 Fahy O, Tsicopoulos A, Hammad H, Pestel J, Tonnel AB, Wallaert B. Effects of diesel organic extracts on chemokine production by peripheral blood mononuclear cells. J Allergy Clin Immunol 1999; 103: 1115-1124.

17 Yanagisawa $\mathrm{R}$, Takano $\mathrm{H}$, Inoue $\mathrm{K}$, et al. Components of diesel exhaust particles differentially affect Th1/Th2 response in a murine model of allergic airway inflammation. Clin Exp Allergy 2006; 36: 386-395.

18 Schuetzle D, Lee FS, Prater TJ. The identification of polynuclear aromatic hydrocarbon (PAH) derivatives in mutagenic fractions of diesel particulate extracts. Int $J$ Environ Anal Chem 1981; 9: 93-144.

19 Schuetzle D. Sampling of vehicle emissions for chemical analysis and biological testing. Environ Health Perspect 1983; 47: 65-80.

20 Monks TJ, Hanzlik RP, Cohen GM, Ross D, Graham DG. Quinone chemistry and toxicity. Toxicol Appl Pharmacol 1992; 112: 2-16.

21 O'Brien PJ. Molecular mechanisms of quinone cytotoxicity. Chem Biol Interact 1991; 80: 1-41.

22 Bolton JL, Trush MA, Penning TM, Dryhurst G, Monks TJ. Role of quinones in toxicology. Chem Res Toxicol 2000; 13: 135-160.

23 Cho A, Di Stefano E, Ying Y, et al. Determination of four quinones in diesel exhaust particles, SRM 1649a, and atmospheric PM2.5. Aerosol Sci Technol 2004; 38: Suppl. 1, 68-81.

24 Hiyoshi $\mathrm{K}$, Takano $\mathrm{H}$, Inoue $\mathrm{K}$, et al. Effects of a single intratracheal administration of phenanthraquinone on murine lung. J Appl Toxicol 2005; 25: 47-51.

25 Hiyoshi K, Takano H, Inoue KI, et al. Effects of phenanthraquinone on allergic airway inflammation in mice. Clin Exp Allergy 2005; 35: 1243-1248.
26 Kumagai Y, Taira J, Sagai M. Apparent inhibition of superoxide dismutase activity in vitro by diesel exhaust particles. Free Radic Biol Med 1995; 18: 365-371.

27 Yanagisawa R, Takano $\mathrm{H}$, Inoue $\mathrm{K}$, et al. Enhancement of acute lung injury related to bacterial endotoxin by components of diesel exhaust particles. Thorax 2003; 58: 605-612.

28 Vouk VB, Piver WT. Metallic elements in fossil fuel combustion products: amounts and form of emissions and evaluation of carcinogenicity and mutagenicity. Environ Health Perspect 1983; 47: 201-225.

29 International Agency for Research on Cancer. IARC monographs on the evaluation of carcinogenic risks to humans: diesel and gasoline exhaust and some nitroarenes. Lyon, IARC, 1989.

30 Quackenbush EJ, Wershil BK, Aguirre V, GutierrezRamos JC. Eotaxin modulates myelopoiesis and mast cell development from embryonic hematopoietic progenitors. Blood 1998; 92: 1887-1897.

31 Humbles AA, Conroy DM, Marleau S, et al. Kinetics of eotaxin generation and its relationship to eosinophil accumulation in allergic airways disease: analysis in a guinea pig model in vivo. J Exp Med 1997; 186: 601-612.

32 Penny LA. Monocyte chemoattractant protein 1 in luteolysis. Rev Reprod 2000; 5: 63-66.

33 Conti P, DiGioacchino M. MCP-1 and RANTES are mediators of acute and chronic inflammation. Allergy Asthma Proc 2001; 22: 133-137.

34 Erger RA, Casale TB. Interleukin-8 plays a significant role in IgE-mediated lung inflammation. Eur Respir J 1998; 11: 299-305.

35 Teran LM, Campos MG, Begishvilli BT, et al. Identification of neutrophil chemotactic factors in bronchoalveolar lavage fluid of asthmatic patients. Clin Exp Allergy 1997; 27: 396-405.

36 Vargaftig BB, Singer M. Leukotrienes, IL-13, and chemokines cooperate to induce BHR and mucus in allergic mouse lungs. Am J Physiol Lung Cell Mol Physiol 2003; 284: L260-L269.

37 Kaneko M, Swanson MC, Gleich GJ, Kita H. Allergenspecific IgG1 and IgG3 through Fc $\gamma$ RII induce eosinophil degranulation. J Clin Invest 1995; 95: 2813-2821.

38 Durham SR, Lee $\mathrm{TH}$, Cromwell $\mathrm{O}$, et al. Immunologic studies in allergen-induced late-phase asthmatic reactions. J Allergy Clin Immunol 1984; 74: 49-60.

39 Martin JG, Duguet A, Eidelman DH. The contribution of airway smooth muscle to airway narrowing and airway hyperresponsiveness in disease. Eur Respir J 2000; 16: 349-354.

40 Morcillo EJ, Cortijo J. Mucus and MUC in asthma. Curr Opin Pulm Med 2006; 12: 1-6. 